Research Paper

\title{
Serum Hyperglycemia Might Be Not Related to Fat Composition of Diet and Vegetable Composition of Diet Might Improve Sugar Control in Taiwanese Diabetic Subjects
}

\author{
Yi-Cheng Hou'1, Ya-Lin Chang1, Shi-Ching Kuo ${ }^{1}$, Chih-Fan Chiang1, Cheng-Yang Chiang1, Yu-Fang Lin'1,

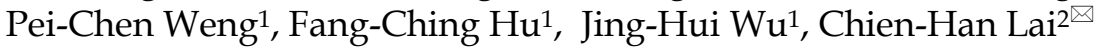 \\ 1. Department of Nutrition, Taipei Tzu Chi Hospital, Buddhist Tzu Chi Medical Foundation, New Taipei City, Taiwan; \\ 2. Department of Psychiatry, Cheng Hsin General Hospital, Taipei, Taiwan. \\ $\triangle$ Corresponding author: Chien-Han Lai, M.D., MSc. Department of Psychiatry, Cheng Hsin General Hospital, No.45, Cheng Hsin St., \\ Pai-Tou Destrict , Taipei City, Taiwan, ROC. Telephone: +886 228264400 ext 3502 Fax: +886 228264570 E-mail: t122336257@yahoo.com.tw. \\ (c) Ivyspring International Publisher. This is an open-access article distributed under the terms of the Creative Commons License (http://creativecommons.org/ \\ licenses/by-nc-nd/3.0/). Reproduction is permitted for personal, noncommercial use, provided that the article is in whole, unmodified, and properly cited.
}

Received: 2013.II.18; Accepted: 2014.03.1I; Published: 2014.03.29

\begin{abstract}
Objective: This is an Asian study, which was designed to examine the correlations between biochemical data and food composition of diabetic patients in Taiwan. Methods: One hundred and seventy Taiwanese diabetic patients were enrolled. The correlations between biochemical data and diet composition (from 24-hour recall of intake food) of these patients were explored (Spearman correlation, $p<0.05$ ). Diet components were also correlated with each other to show diet characteristics of diabetic patients in Taiwan. Linear regression was also performed for the significantly correlated groups to estimate possible impacts from diet composition to biochemical data. Results: Postprandial serum glucose level was negatively correlated with fat percentage of diet, intake amount of polyunsaturated fatty acid and fiber diet composition. Hemoglobin Alc was negatively correlated with fat diet, polyunsaturated fatty acid and vegetable diet. Fat composition, calorie percentage accounted by polyunsaturated fatty acid and monounsaturated fatty acid in diet seemed to be negatively correlated with sugar percentage of diet and positively correlated with vegetable and fiber composition of diet. Linear regression showed that intake amount of polyunsaturated fatty acid, calorie percentage accounted by polyunsaturated fatty acid, fat percentage of diet, vegetable composition of diet would predict lower hemoglobin AIc and postprandial blood sugar. Besides, higher percentage of fat diet composition could predict higher percentage of vegetable diet composition in Taiwanese diabetic patients. Conclusion: Fat diet might not elevate serum glucose. Vegetable diet and polyunsaturated fatty acid diet composition might be correlated with better sugar control in Taiwanese diabetic patients.
\end{abstract}

Key words: fat; vegetable; polyunsaturated fatty acid; hemoglobin A1c; Type II diabetes mellitus.

\section{Introduction}

Type II diabetes mellitus (DM) is a chronic illness affecting around $6 \%$ of world population with incidence of 0.13 to $10 / 100,000 / y r$ in Asia. Type II DM is usually influenced by environmental and genetic factors (1). Within environmental factors, diet is also important for pathogenesis of DM and glycemic diet will exacerbate the glucose control (2). Besides, there are several studies showing the connection between fat composition of diet and serum glucose in DM. Perez-Martinez et al. suggested that Mediterranean 
diet, which is rich in monounsaturated fatty acid (MUFA), might prevent exacerbations of DM and protect cardiovascular system (3). Pohl et al. also found that lower carbohydrate and higher MUFA composition of diet might reduce daily insulin dose, fasting blood glucose and hemoglobin A1c in patients with type II DM. It might suggest that higher MUFA diet composition might improve glycemic control (4). Nettleton and Katz reported that polyunsaturated fatty acid (PUFA) might reduce the rate of conversion from impaired glucose intolerance to type II DM in overweight persons (5). The fat composition of diet is also related to glycemic control of DM. Barnard et al. ever proposed that low-fat vegan diet would reduce hemoglobin A1c, plasma lipid concentration and urinary albumin in patients with type II DM, which might be related to loss of body weight after eating low-fat vegan diet (6). However, there are different findings in an Asian study of elderly patients with type II DM. They found that patients with better control of hemoglobin A1c might depend more on protein or fat than carbohydrate in diet composition. Besides, levels of hemoglobin A1c seemed to be negatively correlated with intake amounts of protein or fat (7). From these studies, fat or unsaturated fatty acids might play a role in the glycemic control in patients with type II DM.

Apart from fat composition of diet, the vegetable content is another possible factor which might serum glucose of patients with type II DM. In Barnard et al's study, they found that low-fat vegan diet would decrease more body weight, cholesterol, serum glucose, hemoglobin A1c and urinary albumin than diet recommended by American Diabetes Association (6). In their subsequent randomized controlled trial of low-fat vegan diet in patients with type II DM, the effects of decreasing hemoglobin A1c and cholesterol were still observed. These results suggest that low-fat vegan diet can provide clinical benefits for controlling glycemia and plasma lipid in diabetic patients (8). Another study of plant-based diet also found similar findings and the diet might provide more intake of fiber, total vitamin A, K, C, several micronutrients and folate. This plant-based diet also improved hemoglobin A1c and body weight (9). These studies suggested that vegetable diet might be helpful to decrease serum glucose in patients with DM.

According to above literatures, we hypothesized that different composition of diet might be associated with serum glucose or hemoglobin A1c in patients with type II DM. Within these kinds of diet composition, vegetable, PUFA, MUFA, or fat might influence or predict serum glucose or hemoglobin A1c level.

\section{Materials and Method}

\section{Participating subjects}

This project was a cross-sectional study and was approved by Institute of Review Board, Buddhist Tzu Chi General Hospital Taipei Branch. The participating patients were recruited from consultation clinics of dietitian located at Buddhist Tzu Chi General Hospital Taipei Branch. All participating subjects signed informed consent and agreed to join this project. We included the patients with type II DM and without the following exclusion conditions: gestational diabetes, renal insufficiency, cancer, myocardial infarction, stroke, amputation, steroid use, operation within 4 weeks and dietitian intervention within 6 months. Demographic data, such as body weight, body height, body mass index (BMI), diastolic blood pressure, systolic blood pressure, age, gender and educational level, were all recorded. All patients received dietary assessment (24-hour recall of food intake), which was done as the following procedures. During each enrolled patient visit, the research dietitians obtained daily nutrient intake by asking patients to recall the consuming foods within previous 24 hours, a routine method of inquiry used in clinical agents of Taiwan. Nutrient intake was analyzed by nutrient analysis software (Nutritionist Edition, Enhancement version 2002, Taichung, Taiwan) (10). The analysis software was developed according to the database for Taiwan Area Nutritive Food Composition Factors, which was published and announced by Department of Health, Exeuctive Yuan, Taiwan. It could help us survey their eating habits and composition of their diets, such as amounts of fat, sugar, vegetable, meat, fish, egg, bean, milk, oil, total calorie, sugar $\%$, fat $\%$, MUFA $\%$, PUFA \%, fiber\%, and correct rate for answering the above questions. Besides, they received blood draw in the morning (no oral intake of food for at least 8 hours), which was aimed to examine the following biochemical parameters: (1) hemoglobin A1c, preprandial and postprandial blood sugar (2) triglyceride, cholesterol, high density lipoprotein (HDL), low density lipoprotein (LDL), alanine aminotransferase (ALT), blood urea nitrogen (BUN), creatinine (CRE), uric acid. (3) urinary microalbumin. At last, the concurrent medications of all patients were also recorded, such as oral hypo-glycemic agent (OHA), oral hypo-lipidemic agent, antihypertensive medications and insulin. The compliance of these patients towards medications was evaluated by inquiring the patients about frequency of medications, appearances and shapes of oral medication and insulin injection formula, side effects of oral medications or insulin injection. Only the patients with at least fair compliance (at least $75 \%$ correct answer rates of the above questions 
and regular use of prescribed medications) would be enrolled into this study.

\section{Statistical analysis}

The correlations between biochemical data and diet composition from food questionnaire (amounts of fat, sugar, vegetable, meat, fish, egg, bean, milk, oil, total calorie, sugar $\%$, fat \%, MUFA $\%$, PUFA $\%$, fiber $\%$ ) were estimated (Spearman correlation, $\mathrm{p}<$ 0.05). Diet composition was also correlated with each other to show diet characteristics of diabetic patients in Taiwan. Linear regression was also performed with adjustments of age, gender, BMI, the kinds and doses of OHA and insulin for significantly correlated groups to estimate the impacts from different composition of diet to biochemical data. Besides, linear regression analysis was used for the correlation between different diet composition with adjustments of age, gender and BMI. The results of linear regression could help us confirm that the amounts of diet composition would predict the level of biochemical data and diet composition. All the above correlations and linear regression were analyzed by SPSS software version 12 (SPSS Inc, Chicago). Because genders may cause changes in the biochemical results, so the results of both sexes were also evaluated separately.

\section{Results}

\section{Demographic data of participating subjects}

One hundred and seventy patients $(56.13 \pm 11.07$ years old, 84 females and 86 males, onset since 9.99 \pm 4.88years ago) were enrolled into this study. The BMI was $26.19 \pm 4.57$, body weight was $69.15 \pm 12.28 \mathrm{~kg}$, body height was $162.18 \pm 8.67 \mathrm{~cm}$, systolic blood pressure was $130.62 \pm 16.52 \mathrm{mmHg}$ and diastolic blood pressure was $82.93 \pm 10.74 \mathrm{mmHg}$. Hemoglobin A1c level was $8.37 \pm 2.14$ [95\% confidence interval (CI): 8.02-8.72], preprandial glucose level was 172.10 $\pm 68.75 \mathrm{mg} / \mathrm{dl}$ (95\% CI: $161.12-183.09 \mathrm{mg} / \mathrm{dl}$ ) and postprandial glucose level was $237.60 \pm 106.07 \mathrm{mg} / \mathrm{dl}$ (95\% CI: $214.72-260.48 \mathrm{mg} / \mathrm{dl}$ ) (Table 1). All these patients received OHA or insulin treatment. 53 patients $(31.17 \%)$ also received oral hypolipidemic treatment and 48 patients $(28.23 \%)$ also received antihypertensive medications.

\section{Correlation results}

Postprandial serum glucose level was negatively correlated with fat percentage of diet, intake amount of PUFA and fiber diet composition. The serum level of hemoglobin A1c was negatively correlated with fat percentage of diet, calorie percentage accounted by PUFA and vegetable composition of diet (Table 2). Intake amount of fat, PUFA and MUFA in diet com- position seemed to be negatively correlated with sugar percentage of diet and positively correlated with intake amount of vegetable and fiber in diet composition (Table 3). For different genders, we still found similar correlated patterns in separate analysis of both genders. For female patients, postprandial serum glucose level was also negatively correlated with fat percentage of diet and intake amount of PUFA, but not fiber diet composition. The serum level of hemoglobin A1c was negatively correlated with fat percentage of diet, calorie percentage accounted by PUFA and vegetable composition of diet. For male patients, postprandial serum glucose level was also negatively correlated with fat percentage of diet and intake amount of PUFA, but not fiber diet composition. The serum level of hemoglobin A1c was negatively correlated with fat percentage of diet, calorie percentage accounted by PUFA and vegetable composition of diet. The correlations between fat, vegetables and sugar percentage diets were also similar in separate analysis of both genders.

Table I: Demographic data of patients with type II DM in this study.

\begin{tabular}{ll}
\hline & Patients \\
\hline Number and gender & $\begin{array}{l}170,84 \text { females } \\
\text { and } 86 \text { males }\end{array}$ \\
Age, mean (SD), years old & $56.13 \pm 11.07$ \\
DM duration, mean (SD), years & $9.99 \pm 4.88$ \\
Preprandial glucose, mean (SD), mg/dl & $172.10 \pm 68.75$ \\
Postprandial glucose, mean (SD), mg/dl & $237.60 \pm 106.07$ \\
Hemoglobin A1c, \% & $8.37 \pm 2.14$ \\
BMI, mean (SD) & $26.19 \pm 4.57$ \\
Body weight, mean (SD), kg & $69.15 \pm 12.28$ \\
Body height, mean (SD), cm & $162.18 \pm 8.67$ \\
Systolic blood pressure, mean (SD), mmHg & $130.62 \pm 16.52$ \\
Diastolic blood pressure, mean (SD), mmHg & $82.93(10.74)$ \\
\hline
\end{tabular}

Table 2: Correlation results between different diet composition and biochemistric data of patients with type II DM.

\begin{tabular}{lll}
\hline & $\begin{array}{l}\text { Postprandial } \\
\text { serum glucose } \\
\text { level }\end{array}$ & $\begin{array}{l}\text { serum level of } \\
\text { hemoglobin } \\
\text { A1c }\end{array}$ \\
\hline Fat percentage (Spearman rho r, p) & $-0.305, \mathrm{p}<0.05$ & $-0.181, \mathrm{p}<0.05$ \\
PUFA (Spearman rho $\mathrm{r}, \mathrm{p}$ ) & $-0.354, \mathrm{p}<0.01$ & $-0.241, \mathrm{p}<0.01$ \\
Fiber (Spearman rho, $\mathrm{p}$ ) & $-0.261, \mathrm{p}<0.05$ & $-0.193, \mathrm{p}<0.05$ \\
\hline
\end{tabular}

Table 3: Correlation results between different diet composition of patients with type II DM.

\begin{tabular}{llll}
\hline & $\begin{array}{l}\text { Fat com- } \\
\text { position }\end{array}$ & PUFA & MUFA \\
\hline Sugar percentage (Spearman & -0.601, & -0.489, & -0.674, \\
rho r, $\mathrm{p}$ ) & $\mathrm{p}<0.01$ & $\mathrm{p}<0.01$ & $\mathrm{p}<0.01$ \\
Vegetable (Spearman rho r, $\mathrm{p})$ & 0.181, & 0.310, & Not sig- \\
& $\mathrm{p}<0.05$ & $\mathrm{p}<0.01$ & nificant \\
Fiber (Spearman rho $\mathrm{r}, \mathrm{p})$ & 0.280, & 0.294, & Not sig- \\
& $\mathrm{p}<0.01$ & $\mathrm{p}<0.01$ & nificant \\
\hline
\end{tabular}




\section{Linear regression results}

Intake amount of PUFA (standardized coefficient: $-0.252 ; \mathrm{p}=0.005)$, calorie percentage accounted by PUFA (standardized coefficient: $-0.324 ; \mathrm{p}<0.001$ ), fat percentage of diet (standardized coefficient: -0.203 ; $\mathrm{p}=0.026$ ), vegetable composition of diet (standardized coefficient: $-0.257 ; \mathrm{p}=0.004$ ) could predict serum level of hemoglobin A1c. All these four parameters showed that higher values would predict lower serum level of hemoglobin A1c. All these predictions were corrected by the age, gender, BMI and kinds and doses of OHA and insulin. Higher fat composition of diet would predict higher vegetable composition of diet $(\mathrm{p}<$ $0.05)$, corrected by age, gender and BMI (Table 5). In separate analysis of both genders, calorie percentage accounted by PUFA (standardized coefficient: -0.315 ; $\mathrm{p}=0.021$ ) and vegetable composition of diet (standardized coefficient: $-0.294 ; \mathrm{p}=0.031$ ) of female patients seemed to predict serum level of hemoglobin A1c. Intake amount of PUFA (standardized coefficient:
-0.288; $p=0.019$ ), calorie percentage accounted by PUFA (standardized coefficient: $-0.330 ; p=0.007$ ), fat percentage of diet (standardized coefficient: -0.207 ; $\mathrm{p}=0.036$ ) of male patients seemed also to predict hemoglobin A1c level (Table 6).

\section{Discussion}

In this study, we found that intake amount of PUFA, calorie percentage accounted by PUFA, fat percentage of diet, vegetable composition of diet might predict lower level of hemoglobin A1c. Therefore, it suggested that better glycemic control might be related to fat and vegetable composition of diet. Besides, the patients who consumed more fats seemed to eat more vegetables in their diets. It suggested that higher fat composition of diet might be associated with lower level of hemoglobin A1c due to higher vegetable composition of diet in these patients with type II DM.

Table 4: Correlation results between different diet composition and biochemistric data of patients with type II DM in different genders.

\begin{tabular}{lll}
\hline & postprandial serum glucose level & serum level of hemoglobin A1c \\
\hline Female & & \\
Fat percentage (Spearman rho r, p) & $-0.484, \mathrm{p}<0.05$ & $-0.205, \mathrm{p}<0.05$ \\
PUFA (Spearman rho r, p) & $-0.395, \mathrm{p}<0.05$ & $-0.315, \mathrm{p}<0.05$ \\
Fiber (Spearman rho r, p) & Not significant & Not significant \\
Vegetable & Not significant & $-0.294, \mathrm{p}<0.05$ \\
Male & & \\
Fat percentage (Spearman rho r, p) & $-0.240, \mathrm{p}<0.05$ & $-0.286, \mathrm{p}<0.05$ \\
PUFA (Spearman rho $\mathrm{r}, \mathrm{p}$ ) & $-0.434, \mathrm{p}<0.05$ & $-0.284, \mathrm{p}<0.05$ \\
Fiber (Spearman rho r, $\mathrm{p}$ ) & Not significant & Not significant \\
Vegetable & Not significant & $-0.131, \mathrm{p}<0.05$ \\
\hline
\end{tabular}

Table 5: Linear regression analysis of diet, hemoglobin AIc and postprandial glucose of patients with type II DM.

\begin{tabular}{lllll}
\hline Independent variable & Dependent variable & Unstandardized coefficients & Standardized coefficients & Significance \\
\hline Intake amounts of PUFA & hemoglobin A1c & -0.046 & -0.252 & 0.005 \\
Fat percentage & hemoglobin A1c & -0.050 & -0.203 & 0.026 \\
Vegetable & hemoglobin A1c & -0.321 & -0.257 & 0.004 \\
Calorie percentage accounted by PUFA & Postprandial glucose & -2152.903 & -0.368 & 0.002 \\
Calorie percentage accounted by PUFA & hemoglobin A1c & -36.628 & -0.324 & $<0.001$ \\
Fat & Vegetable & 0.009 & 0.194 & 0.021 \\
\hline
\end{tabular}

Table 6: Linear regression analysis of diet, hemoglobin AIc and postprandial glucose of patients with type II DM in both genders.

\begin{tabular}{|c|c|c|c|c|}
\hline Independent variable & Dependent variable & Unstandardized coefficients & Standardized coefficients & Significance \\
\hline \multicolumn{5}{|l|}{ Female } \\
\hline Vegetable & hemoglobin A1c & -0.376 & -0.294 & 0.031 \\
\hline Calorie percentage accounted by PUFA & hemoglobin A1c & -33.395 & -0.315 & 0.021 \\
\hline Fat & Vegetable & 0.009 & 0.159 & 0.02 \\
\hline \multicolumn{5}{|l|}{ Male } \\
\hline Intake amount of PUFA & hemoglobin A1c & -0.048 & -0.288 & 0.019 \\
\hline Fat percentage & hemoglobin A1c & -0.049 & -0.207 & 0.036 \\
\hline Calorie percentage accounted by PUFA & hemoglobin A1c & -39.331 & -0.330 & 0.007 \\
\hline
\end{tabular}


The findings of PUFA in this study seemed to suggest that PUFA might be relatively important in glycemic control. In a similar Asian study, Ghosh found that lean controls had greater mean level of PUFA than diabetic patients. It suggested that fatty acids and ratios might provide the index for identifying diabetic group (11). This study also supports our findings about the important role of PUFA in Taiwanese subjects with type II DM. PUFA-related diets, such as whole grain products, fish and bilberry, might improve glycemic control through decreasing serum glucose, increasing insulin secretion and glucose disposal. Lankinen et al. suggested that PUFA-related diet may have a beneficial effect to prevent high risk persons from getting type II DM (12). In the animal model of vascular response and insulin resistance, PUFA can reduce vascular complications and improve the phenomenon of insulin-resistance (13). Another study of animal model for PUFA in type II DM also found that PUFA could reduce insulin resistance and increase insulin sensitivity due to increased adiponectin and decreased tumor necrosis factor-alpha in plasma. Besides, PUFA could result in downregulated expression of the lipogenic genes, upregulation of the fatty acid oxidative gene and the energy expenditure-related genes in white adipose tissue (14). These mechanisms might provide the theoretical basis to explain why PUFA could be associated better glycemic control. However, there is still contrary point of view which suggested that PUFA just could lower triglyceride and very low density lipoprotein without significant effect on glycemic control or fasting insulin. PUFA even would raise LDL level even without statistical significance (15). The controversial might need further and well-organized study to clarify this issue.

In this study, MUFA did not predict lower hemoglobin A1c or was not associated with better glycemic control. Brunerova et al. found that MUFA could decrease hemoglobin A1c and serum glucose. They also suggested that diet of higher fat might show mild superiority for improving metabolic parameters in type II DM (16). Due A et al. found that MUFA diet might improve insulin resistance, fasting glucose and lipid profile in non-diabetic obese patients (17). Insulin-resistant patients also seem to be responsive to MUFA diet for improving postprandial glucose, insulin concentration and glucogan-like peptide, which might suggest that MUFA can improve clinical symptoms of type II DM (18). MUFA also can increase glucagons-like peptide by enhancing L cell sensitivity of pancreas (19). Even these studies suggest that MUFA should be associated with lower hemoglobin A1c, there was no such link in this study. The possible reasons might be that the hypoglycemic effect of
MUFA is not so significant as those of PUFA or vegetables in Taiwanese patients. According to Marin et al.'s study, they suggested that insulin sensitivity and glycemic control of type II DM should be determined by the environmental and genetic factors, not just sole factor (20). The relationship between MUFA and hemoglogin A1c or serum glucose might need more studies to be clarified in Asian patients. Besides, underlying genetic differences between different races can be an intrigue issue for further study in the future.

Tonstad et al. found that vegetable-only diet would reduce BMI and provide a substantial potential to protect vegetarians against the risk of type II DM after taking lifestyle characteristics and BMI into account (21). In the vegetarian study of Valachovicova et al., they suggested that vegetarian diet would not cause insulin resistance. In the contrary, long-term vegetarian nutrition could offer beneficial effect in prevention of type II DM and metabolic syndrome due to age independent effects and lower values of insulin resistance (22). Vegetable with high fiber diet also can improve glucose metabolism and increase insulin sensitivity (23). These studies all found similar effects of vegetable-related diets in previous studies about vegan diets in type II DM $(6,8,24)$. Vegetable-related diets can reduce body weights, subcutaneous fat or visceral fat, leptin and oxidative stress. Besides, vegetable-related diets will increase insulin sensitivity, adiponectin, vitamin $C$, superoxide dismutase and reduced glutathione in patients with type II DM (25). In the review of studies about vegetarian and vegan diet in type II DM, vegetable-related diets were superior in glycemic control than traditional diabetes diets. Besides, plant-based diets might influence serum glucose due to the following reasons, such as reduced intake of saturated fats and high-glycemic-index foods, increased intake of dietary fiber and vegetable protein, reduced intramyocellular lipid concentrations, and decreased iron stores. At last, plant-based diets would also improve plasma lipid concentrations and thus show the ability to reverse artherosclerosis progression in DM patients (26).

Out of our expectations, higher fat seemed not so harmful for the glycemic control in patients with type II DM. A meta-analysis of high fat versus low fat diet in type II DM showed that high fat diet might be with lower risk of deteriorations of insulin resistance than low fat diet. Besides, high fat diet would be associated with better lipid profile than low fat diet in type II DM (27). It suggested that higher composition of fat in diet might improve glycemic control of diabetic patients. Apart from the above study, the positive influences of fat diet composition in glycemic control can also be explained by that high fat composition in this study 
might be accounted by relatively higher amounts of PUFA, which could predict lower hemoglobin A1c. However, the separate analysis of both genders showed that only male patients would still have such impacts of fat composition towards hemoglobin A1c. It suggested that female patients might have less hypoglycemic effects of fat diet composition. Besides, higher fat composition of diet predicted higher vegetable composition in this study. As the above results, vegetable could predict lower hemoglobin A1c. This possibility can also help us explain the relationship between glycemic control and fat diet composition. However, this theory just can explain the phenomenon in female patients due to that higher fat composition with higher vegetable composition was just significant in separate analysis of female patients. The association between fat and vegetable composition of diet might represent a special pattern in this group of Taiwanese patients with type II DM. This unique diet pattern could be an intrigue issue for future study.

In the separate analysis of both genders, male and female patients showed similar correlated patterns between diet composition and biochemical data. The correlated patterns were also similar with the results of total population. However, in separate linear regression analysis, male patients showed that higher PUFA and fat diet composition would predict lower hemoglobin A1c. Female patients showed that higher vegetable and PUFA diet composition could predict lower hemoglobin A1c. This is another interesting finding of our study. It might suggest that vegetable factor might be more sensitive in female patients and fat factor would be more sensitive in male patients. Both male and female patients showed similar patterns of PUFA in glycemic control. From the results of separate analysis, we suggest that PUFA might be the most important factor for glycemic control in Taiwanese patients with type II DM.

There are several limitations in our study. First, the compliance of OHA or insulin is a potential confounding factor for our results. However, we just enrolled the patients with at least fair compliance in this study, which might reduce influences from this issue. But lack of quantitative assessment of compliance might limit the interpretations of our results. Second, sample size of this study is relatively small, which might bias our results. Third, use of OHA and insulin would directly influence glycemic control of these patients. Even all these patients used OHA or insulin and the influences of OHA or insulin were adjusted in statistical analysis, the possible effects of OHA and insulin should be noted in this study. A further study with more patients and less dependent on medications might be warranted in the future. Fourth, our study design is cross-sectional, which might be diffi- cult to measure prediction, associations and any causal relationships. Moreover, correlation coefficients as the only outcome measure are not informative and might give only little information about any associations between two measures.

\section{Conclusion}

In this study, we suggested that fat diet might not elevate serum glucose. Vegetable composition in diet and PUFA composition in diet might be correlated with better glycemic control in diabetic patients.

\section{Acknowledgements}

We want to thank for the grant support from Buddhist Tzu-Chi General Hospital, Taipei Branch hospital project TCRD-TPE-100-04. We also acknowledge the help of all dietitians of Department of Nutrition to enroll the patients of this study.

\section{Competing Interests}

The authors have declared that no competing interest exists.

\section{References}

1. Adeghate E, Schattner P, Dunn E. An update on the etiology and epidemiology of diabetes mellitus. Ann N Y Acad Sci. 2006; 1084: 1-29.

2. Marsh K, Barclay A, Colagiuri S, et al. Glycemic index and glycemic load of carbohydrates in the diabetes diet. Curr Diab Rep. 2011; 11: 120-7.

3. Perez-Martinez P, Garcia-Rios A, Delgado-Lista I, et al. Mediterranean diet rich in olive oil and obesity, metabolic syndrome and diabetes mellitus. Curr Pharm Des. 2011; 17: 769-77.

4. Pohl M, Mayr P, Mertl-Roetzer M, et al. Glycaemic control in type II diabetic tube-fed patients with a new enteral formula low in carbohydrates and high in monounsaturated fatty acids: a randomised controlled trial. Eur J Clin Nutr. 2005; 59: 1221-32.

5. Nettleton JA, Katz R. n-3 long-chain polyunsaturated fatty acids in type 2 diabetes: a review. J Am Diet Assoc. 2005; 105: 428-40.

6. Barnard ND, Cohen J, Jenkins DJ, et al. A low-fat vegan diet improves glycemic control and cardiovascular risk factors in a randomized clinical trial in individuals with type 2 diabetes. Diabetes Care. 2006; 29: 1777-83.

7. Woo MH, Park S, Woo JT, et al. A comparative study of diet in good and poor glycemic control groups in elderly patients with type 2 diabetes mellitus. Korean Diabetes J. 2010; 34: 303-11.

8. Barnard ND, Cohen J, Jenkins DJ, et al. A low-fat vegan diet and a conventional diabetes diet in the treatment of type 2 diabetes: a randomized, controlled, 74-wk clinical trial. Am J Clin Nutr. 2009; 89: 1588S-96S.

9. Turner-McGrievy GM, Barnard ND, Cohen J, et al. Changes in nutrient intake and dietary quality among participants with type 2 diabetes following a low-fat vegan diet or a conventional diabetes diet for 22 weeks. J Am Diet Assoc. 2008; 108: 1636-45.

10. Huang MC, Hsu CC, Wang HS, et al. Prospective randomized controlled trial to evaluate effectiveness of registered dietitian-led diabetes management on glycemic and diet control in a primary care setting in Taiwan. Diabetes Care. 2010; 33: 233-9.

11. Ghosh A. Anthropometric, metabolic and dietary fatty acids profiles in lean and obese diabetic Asian Indian subjects. Asia Pac J Clin Nutr. 2006; 15: 189-95.

12. Lankinen $M, S c h w a b U$, Kolehmainen $M$, et al. Whole grain products, fish and bilberries alter glucose and lipid metabolism in a randomized, controlled trial: the Sysdimet study. PLoS ONE. 2011; 6: e22646.

13. Mustad VA, Demichele S, Huang YS, et al. Differential effects of $n-3$ polyunsaturated fatty acids on metabolic control and vascular reactivity in the type 2 diabetic ob/ob mouse. Metabolism. 2006; 55: 1365-74.

14. Yang $\mathrm{ZH}$, Miyahara $\mathrm{H}$, Takemura $\mathrm{S}$, et al. Dietary saury oil reduces hyperglycemia and hyperlipidemia in diabetic KKAy mice and in diet-induced obese C57BL/6J mice by altering gene expression. Lipids. 2011; 46: 425-34.

15. Hartweg J, Perera R, Montori V, et al. Omega-3 polyunsaturated fatty acids (PUFA) for type 2 diabetes mellitus. Cochrane Database Syst Rev. 2008: CD003205.

16. Brunerova L, Smejkalova V, Potockova J, et al. A comparison of the influence of a high-fat diet enriched in monounsaturated fatty acids and conventional 
diet on weight loss and metabolic parameters in obese non-diabetic and Type 2 diabetic patients. Diabet Med. 2007; 24: 533-40.

17. Due A, Larsen $\mathrm{TM}, \mathrm{Mu} \mathrm{H}$, et al. Comparison of 3 ad libitum diets for weight-loss maintenance, risk of cardiovascular disease, and diabetes: a 6-mo randomized, controlled trial. Am J Clin Nutr. 2008; 88: 1232-41.

18. Paniagua JA, de la Sacristana AG, Sanchez E, et al. A MUFA-rich diet improves posprandial glucose, lipid and GLP-1 responses in insulin-resistant subjects. J Am Coll Nutr. 2007; 26: 434-44.

19. Rocca AS, LaGreca J, Kalitsky J, et al. Monounsaturated fatty acid diets improve glycemic tolerance through increased secretion of glucagon-like peptide-1. Endocrinology. 2001; 142: 1148-55.

20. Marin C, Perez-Martinez P, Delgado-Lista J, et al. The insulin sensitivity response is determined by the interaction between the G972R polymorphism of the insulin receptor substrate 1 gene and dietary fat. Mol Nutr Food Res. 2011; 55: 328-35.

21. Tonstad S, Butler T, Yan R, et al. Type of vegetarian diet, body weight, and prevalence of type 2 diabetes. Diabetes Care. 2009; 32: 791-6.

22. Valachovicova M, Krajcovicova-Kudlackova M, Blazicek P, et al. No evidence of insulin resistance in normal weight vegetarians. A case control study. Eur J Nutr. 2006; 45: 52-4.

23. Wolfram T, Ismail-Beigi F. Efficacy of high-fiber diets in the management of type 2 diabetes mellitus. Endocr Pract. 2011; 17: 132-42.

24. Barnard ND, Gloede L, Cohen J, et al. A low-fat vegan diet elicits greater macronutrient changes, but is comparable in adherence and acceptability, compared with a more conventional diabetes diet among individuals with type 2 diabetes. J Am Diet Assoc. 2009; 109: 263-72.

25. Kahleova H, Matoulek M, Malinska H, et al. Vegetarian diet improves insulin resistance and oxidative stress markers more than conventional diet in subjects with Type 2 diabetes. Diabet Med. 2011; 28: 549-59.

26. Barnard ND, Katcher HI, Jenkins DJ, et al. Vegetarian and vegan diets in type 2 diabetes management. Nutr Rev. 2009; 67: 255-63.

27. Kodama S, Saito K, Tanaka S, et al. Influence of fat and carbohydrate proportions on the metabolic profile in patients with type 2 diabetes: a meta-analysis. Diabetes Care. 2009; 32: 959-65. 